\title{
Role of Extracranial Carotid Duplex and Computed Tomography Perfusion Scanning in Evaluating Perfusion Status of Pericarotid Stenting
}

\author{
Chih-Ming Lin, ${ }^{1}$ Yu-Jun Chang, ${ }^{2}$ Chi-Kuang Liu, ${ }^{3}$ \\ Cheng-Sheng $\mathrm{Yu},{ }^{4}$ and Henry Horng-Shing $\mathrm{Lu}^{4}$ \\ ${ }^{1}$ Stroke Center, Department of Neurology, Changhua Christian Hospital, Changhua and Graduate Institute of \\ Biological Science and Technology, National Chiao Tung University, Hsinchu, Taiwan \\ ${ }^{2}$ Epidemiology and Biostatistics Center, Changhua Christian Hospital, Changhua, Taiwan \\ ${ }^{3}$ Department of Medical Imaging, Changhua Christian Hospital, Changhua, Taiwan \\ ${ }^{4}$ Institute of Statistics, National Chiao Tung University, 1001 Ta Hsueh Road, Hsinchu 30010, Taiwan
}

Correspondence should be addressed to Henry Horng-Shing Lu; hslu@stat.nctu.edu.tw

Received 31 January 2016; Accepted 17 February 2016

Academic Editor: Aristomenis K. Exadaktylos

Copyright (C) 2016 Chih-Ming Lin et al. This is an open access article distributed under the Creative Commons Attribution License, which permits unrestricted use, distribution, and reproduction in any medium, provided the original work is properly cited.

\begin{abstract}
Carotid stenting is an effective treatment of choice in terms of treating ischemic stroke patients with concomitant carotid stenosis. Though computed tomography perfusion scan has been recognized as a standard tool to monitor/follow up this group of patients, not everyone could endure due to underlying medical illness. In contrast, carotid duplex is a noninvasive assessment tool and could track patient clinical condition in real time. In this study we found that "resistance index" of the carotid ultrasound could detect flow changes before and after the stenting procedure, thus having great capacity to replace the role of computed tomography perfusion exam.
\end{abstract}

\section{Introduction}

Stroke is the fourth leading cause of death worldwide [1]. Among its various etiologies, carotid stenosis is the most well observed and accounts for 30 to 35 percent of total ischemic strokes [2]. Stenosis grading exceeding 70\% lumen reduction doubles or triples the risk of stroke and its recurrence. Vascular neurologists have reported that prompt management of carotid stenosis through standard carotid stenting prevents stroke and its recurrence. In the prestenting phase, doctors routinely administer computed tomography (CT) perfusion scans to study the cerebral blood perfusion condition in the bilateral cerebral hemispheres.

Although CT perfusion scanning has several advantages and has been recognized as a standard tool for assessing cerebral perfusion in patients undergoing stenting, not all patients can tolerate it. This neuroimaging tool entails the use of a contrast medium, which is hazardous to patients, especially those with impaired renal function.
Extracranial carotid ultrasounding is noninvasive and requires no contrast mediums. Investigation and validation of the role of ultrasound in evaluating flow conditions in pre- and poststenting phases are yet to be reported in the literature. This study utilizes a clinical parameter-resistance index (RI) - generated through carotid ultrasound exams and CT perfusion scans to investigate whether flow changes can be simultaneously observed in these two examinations.

\section{Materials and Methods}

2.1. Patient Identification. We consecutively recruited 15 patients scheduled to undergo carotid stenting. The patients were admitted to our outpatient clinics and emergency departments or transferred from our branch hospital; all patients had been hospitalized for examination and treatment. We included patients with age $\geq 18$ years, with an initial ischemic or recurrent strokes, with angiographic evidence of $>70 \%$ carotid stenosis, with no other etiology 
of stroke that could explain the index event, and with no evidence of recurrent stroke during the study period and followed up at least 6 months after the stenting treatment. Exclusion criteria were patients with cerebral hemorrhage, cerebral arteriovenous malformations, aneurysms, and bilateral moderate-severe carotid stenosis, and less than 6 months' follow-ups. The enrolled patients were hospitalized for medical treatments along with baseline biochemistry work-ups. The ischemic stroke was confirmed by the diffusion weighted sequence of magnetic resonance imaging. The diagnostic digital subtraction angiography (DSA) was arranged during the hospitalization to gauge the degree of the carotid stenosis. The patients were stented one month after the index episode (stroke event). The participants were administered extracranial carotid ultrasound and CT angiography/perfusion (CTA/P) scanning simultaneously before carotid stenting. The above mentioned examinations were repeated simultaneously one month after stenting. This study was approved by the Institutional Review Board of Changhua Christian Hospital.

2.2. Baseline Clinical Characteristics. Baseline demographic data and clinical characteristics were collected including age, gender, and body mass index (BMI); baseline biochemistry data were collected on admission, such as low density lipoprotein (LDL) and glycated hemoglobin (HbAlc) levels and evidence or any major past history. Performance in activities of daily living was measured before stenting with the Barthel Index.

2.3. Cervical Carotid Ultrasound Examination. Cervical carotid artery examination was performed in our ultrasonography laboratory by using a Philips iE33 7-Mhz linear transducer. Patients slightly tilted their head contralaterally, and the transducer was placed on their necks. First, crosssectional B-mode scanning and longitudinal screening were performed to identify and confirm intraluminal plaques, respectively. Peak systolic velocity (PSV), end diastolic velocity (EDV), and resistance index (RI) of the CCA, internal carotid artery (ICA), and external carotid artery (ECA) were measured. RI is given by PSV - EDV/PSV. The degree of carotid stenosis was calculated using the European Carotid Surgery Trial method [3].

2.4. Computed Tomography Angiography/Perfusion Scan (CTA/P Imaging). CTA examinations were performed using a second-generation dual-source CT scanner (SOMATOM Definition Flash, Siemens Healthcare, Forchheim, Germany). Perfusion data sets were postprocessed using a Siemens Multimodality Workplace Workstation (Siemens Medical, Germany), which calculated mean transit time (MTT), cerebral blood volume (CBV), cerebral blood flow (CBF), and time to peak (TTP). The arterial input and venous outflow curves were analyzed to ensure data set completeness. The CTP parameters are defined as follows:

(1) dMTT: ipsilateral MTT - contralateral MTT.

(2) MTT ratio: ipsilateral MTT/contralateral MTT.
(3) MTT index: (ipsilateral MTT - contralateral MTT)/ contralateral MTT.

(4) dCBV: ipsilateral CBV - contralateral CBV.

(5) CBV ratio: ipsilateral CBV/contralateral CBV.

(6) CBV index: (ipsilateral CBV - contralateral CBV)/ contralateral CBV.

(7) dCBF: ipsilateral CBF - contralateral CBF.

(8) $\mathrm{CBF}$ ratio: ipsilateral $\mathrm{CBF} /$ contralateral $\mathrm{CBF}$.

(9) $\mathrm{CBF}$ index: (ipsilateral $\mathrm{CBF}$ - contralateral CBF)/contralateral CBF.

(10) dTTP: ipsilateral TTP - contralateral TTP.

(11) TTP ratio: ipsilateral TTP/contralateral TTP.

(12) TTP index: (ipsilateral TTP - contralateral TTP)/ contralateral TTP.

2.5. Magnetic Resonance Imaging and Angiography (MRI/A). Structural and functional MR imaging and angiographic examinations were performed using a 3-T (Magnetom Verio, Siemens Healthcare, USA) or a 1.5-T imager (Magnetom Aera, Siemens Healthcare) with a cervical coil. Standard protocol to evaluate a stroke including axial DWI, apparent diffusion coefficient, and fluid-attenuated inversion-recovery sequences was followed. Contrast-enhanced MR angiography was not routinely performed.

2.6. Digital Subtraction Angiography (DSA) and Stenting. Biplanar intra-arterial DSA was performed using a biplanar flap panel rotational angiography unit (Axiom Artis Zee, Siemens Healthcare) with an image intensifier matrix of $1024 \times 1024$ pixels and a final pixel size of $0.37 \mathrm{~mm}$. A selfexpandable carotid wallstent $(7 \mathrm{~mm} \times 30 \mathrm{~mm})$ was delivered coaxially through the guiding catheter into the stenotic area (Figure 1).

2.7. Statistical Analyses. Continuous variables are presented as mean \pm standard deviation (SD), median, percentile, minimal, and maximal values. Categorical variables are presented as numbers and percentages. Pre- and poststenting CT perfusion and carotid ultrasound variables were compared using Wilcoxon signed ranks test. $P<0.05$ was considered statistically significant. All statistical analyses were performed using SPSS for Windows (Version 16.0, SPSS Inc., Chicago, IL, USA).

\section{Results}

The baseline clinical characteristics of the 15 patients are summarized in Tables 1 and 2. Tables 3 and 4 report the flow changes reflected by the parameters of $\mathrm{CT}$ perfusion and carotid ultrasound examinations.

Ipsilateral mean values of MTT, CBV, and TTP decreased after stenting, whereas CBF increased slightly. The majority of patients exhibited decreased MTT, CBV, and TTP. Preand posttreatment TTP values differed significantly $(P=$ $0.031,<0.05)$. Contralateral MTT, CBV, and TTP decreased, 


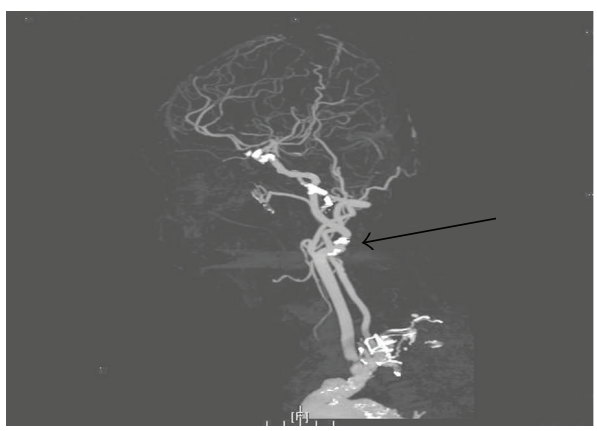

(a)

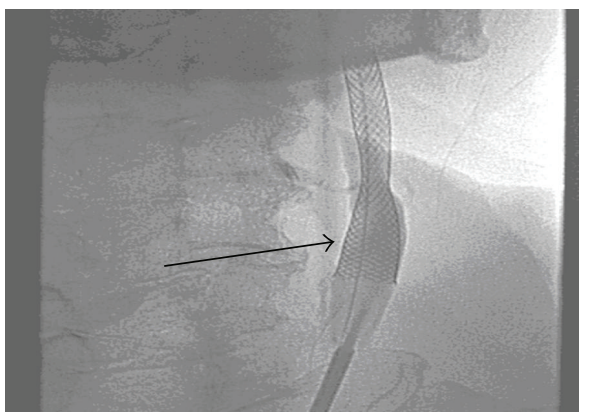

(c)

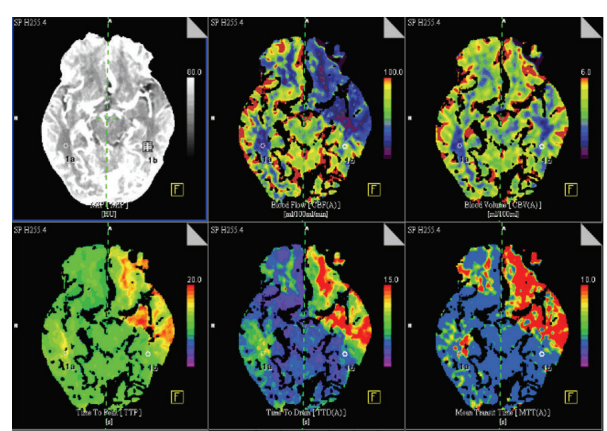

(b)

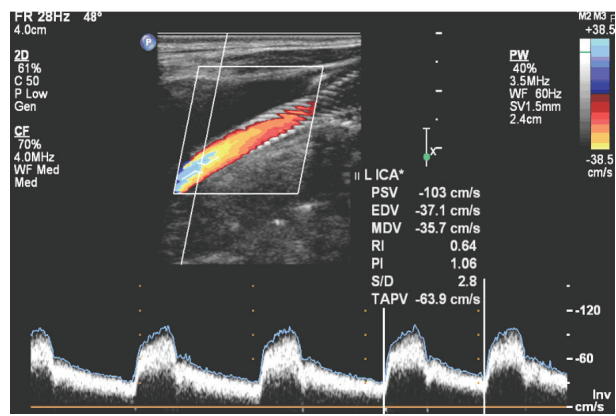

(d)

FIGURE 1: An example of left side severe internal carotid artery stenosis undergoing carotid stenting treatment. An 85-year-old male patient presented with right side hemiparesis and aphasia 24 hours before admission to our neurology ward. (i) (a) denotes CTA scan showing $>70 \%$ lumen reduction of left side proximal internal carotid artery and distal part of common carotid artery (black arrow). (ii) (b) shows the CT perfusion scan that suggests decreased blood perfusion to the left cerebral hemisphere indicating critical blood flow demand compared to the right side cerebral hemisphere. (iii) (c) presents the poststenting status with umbrella device that prevent embolus distal migration causing major subsequent stroke event (black arrow). (iv) (d) displays the follow-up cervical carotid ultrasound of normalized flow profile.

TABLE 1: Baseline patient features.

\begin{tabular}{|c|c|c|c|c|c|c|c|}
\hline$(n=15)$ & Mean & $\mathrm{SD}$ & Median & Percentile 25 & Percentile 75 & Min & Max \\
\hline Age & 66.47 & 9.59 & 69.00 & 56.00 & 73.00 & 47.00 & 79.00 \\
\hline Grade of stenosis & 0.71 & 0.10 & 0.74 & 0.67 & 0.78 & 0.53 & 0.84 \\
\hline SBP & 137.27 & 10.73 & 138.00 & 128.00 & 149.00 & 118.00 & 155.00 \\
\hline DBP & 77.47 & 8.33 & 80.00 & 74.00 & 84.00 & 62.00 & 90.00 \\
\hline Weight & 64.36 & 10.22 & 63.50 & 55.00 & 68.50 & 48.00 & 86.00 \\
\hline Height & 163.47 & 8.33 & 165.00 & 162.00 & 170.00 & 144.00 & 172.00 \\
\hline BMI & 24.09 & 3.36 & 23.56 & 20.78 & 28.20 & 18.34 & 29.07 \\
\hline Barthel & 87.50 & 10.14 & 87.50 & 80.00 & 100.00 & 75.00 & 100.00 \\
\hline LDL & 121.14 & 29.41 & 121.00 & 97.90 & 134.00 & 86.40 & 175.00 \\
\hline HbAlc & 6.94 & 1.89 & 6.65 & 5.70 & 7.30 & 5.30 & 12.60 \\
\hline Ac sugar & 119.67 & 46.18 & 96.00 & 94.00 & 128.00 & 80.00 & 227.00 \\
\hline Uric acid & 6.25 & 1.43 & 6.35 & 5.40 & 7.00 & 4.10 & 9.20 \\
\hline
\end{tabular}

Note. Min, minimum; Max, maximum; SBP, systolic blood pressure; DBP, diastolic blood pressure; BMI, body mass index; LDL, low density lipoprotein; SD, standard deviation.

HbAlc, glycated hemoglobin; AC sugar, fasting blood sugar.

whereas the mean CBF increased slightly. The ratio of patients exhibiting decreased and increased contralateral MTT, CBV, $\mathrm{CBF}$, and TTP values is approximately 1 (Table 3 ).

Ipsilateral RI analyses showed that mean CCA RI reduced from 0.78 to 0.75 , and most patients exhibited decreased CCA RIs. By contrast, mean ICA RI increased from 0.62 to
0.69 , and most patients exhibited increased ICA RIs. Mean ECA RI decreased from 0.84 to 0.80 ; there are five and six patients, demonstrating decreased and increased ECA RIs, respectively (Table 3 ).

Contralateral RI analyses revealed that CCA, ICA, and ECA RIs increased after stenting, with most patients showing 
TABLE 2: Baseline patient demographics.

\begin{tabular}{lcc}
\hline & $N$ & $\%$ \\
\hline Gender & 2 & \\
$\quad$ Female & 13 & 13.3 \\
$\quad$ Male & & 86.7 \\
Diabetes mellitus & 6 & \\
$\quad$ No & 9 & 40.0 \\
$\quad$ Yes & & 60.0 \\
Hypertension & 3 & \\
$\quad$ No & 12 & 20.0 \\
$\quad$ Yes & & 80.0 \\
Hyperlipidemia & 4 & 26.7 \\
$\quad$ No & 11 & 73.3 \\
$\quad$ Yes & & \\
Prior stroke & 8 & 53.3 \\
No & 7 & 46.7 \\
$\quad$ Yes & &
\end{tabular}

increased mean CCA and ICA values; CCA RI increased from 0.73 to 0.77 , ICA RI increased from 0.67 to 0.70 , and ECA increased from 0.87 to 0.88 (Table 3 ).

The mean values of all other $12 \mathrm{CT}$ perfusion scan parameters (Table 4) decreased after stenting. The majority of patients showed decreased values. Among the 12 parameters, MTT ratio, MTT index, and TTP ratio decreased significantly $(P=0.013,0.039$, and $0.017,<0.05)$.

\section{Discussion}

Stroke is the fourth leading cause of death worldwide $[1,2]$, and, among its various etiologies, carotid stenosis accounts for the majority of strokes. Carotid stenting can effectively prevent stroke and its recurrence.

CT perfusion is a standard assessment routinely performed before carotid stenting because it provides vivid color images and several parameters that clarify the ipsilateral and contralateral cerebral hemispheres perfusion status, as well as identifying stroke location [4-9]. However, not all patients can undergo this assessment tool. This is because they entail the use of contrast mediums, which is hazardous to patients with impaired renal function. In addition, CT perfusion administration is restricted to medical facilities, thus requiring patients to visit the facility. Moreover, it requires multiple operators, making it time intensive and expensive for patients.

By contrast, carotid duplex is a mobile, single-operator, contrast-medium-free, and inexpensive examination. Although no studies have examined the role of RI in evaluating cerebral perfusion status after carotid stenting, RI has been widely used in nephrology. Derchi et al. tested RI in patients with renal dysfunction and reported that the risk of renal impairment increased twofold when renal RI was $>0.63$ [10]. In addition, RI is effective in predicting kidney transplant outcomes [11-13].

CT perfusion, by convention, is used to assess the cerebral artery perfusion status. The improvement of cerebral blood



FIGURE 2: RI and flow changes of the patients receiving carotid stenting treatment. RI changes within ipsilateral and contralateral carotid systems. Red color denotes increased blood flow and blue color decreased flow.

perfusion after stenting treatment can be indicated by a reduced MTT and an incased CBF within the ipsilateral carotid system $[14,15]$. Our study is in accordance with these statements. The findings of MTT and CBF over the contralateral carotid system in the published papers $[2,16]$, however, have not come to general consensus. Our study shows that the MTT and CBF values decreased after treatment. Moreover, the absolute difference, ratio, and index values of bilateral cerebral hemispheres are also calculated. The results show a drop in values, particularly within the MTT ratio, MTT index, and TTP ratio.

RI, a clinical parameter generated from carotid ultrasounding, represents the general downstream blood vascular bed resistance level [17]. RI > 0.75 denotes increased downstream vascular bed, which can be due to various factors, including obstructions. Because CCA and ICA supply the majority of blood to the intracranial hemispheres, their RI values are lower than that of ECA in normal circumstances (CCA and ICA < 0.75, ECA > 0.75).

In our study, ipsilateral mean CCA RI values decreased from 0.78 to 0.75 after carotid treatment, indicating that ICA flow smoothened and resistance level decreased after treatment, subsequently drawing more blood to perfuse the same side of the cerebral hemisphere. The mean ipsilateral ICA RI value, however, rose from 0.62 to 0.69 , elucidating the vasoconstriction mechanism after upstream dilation. Nevertheless, ipsilateral CCA and ICA returned to their normal values $(<0.75)$. Conversely, on the contralateral side of the carotid system, mean RI rose in all carotid arteries (CCA, CA, and ECA), which explains the relatively low blood flow to the contralateral side of the vasculature because most of the blood was supplied to the ipsilateral side after stenting (Figure 2).

Our study has a few limitations. First, with only 15 patients, the sample size is small; the results must be 


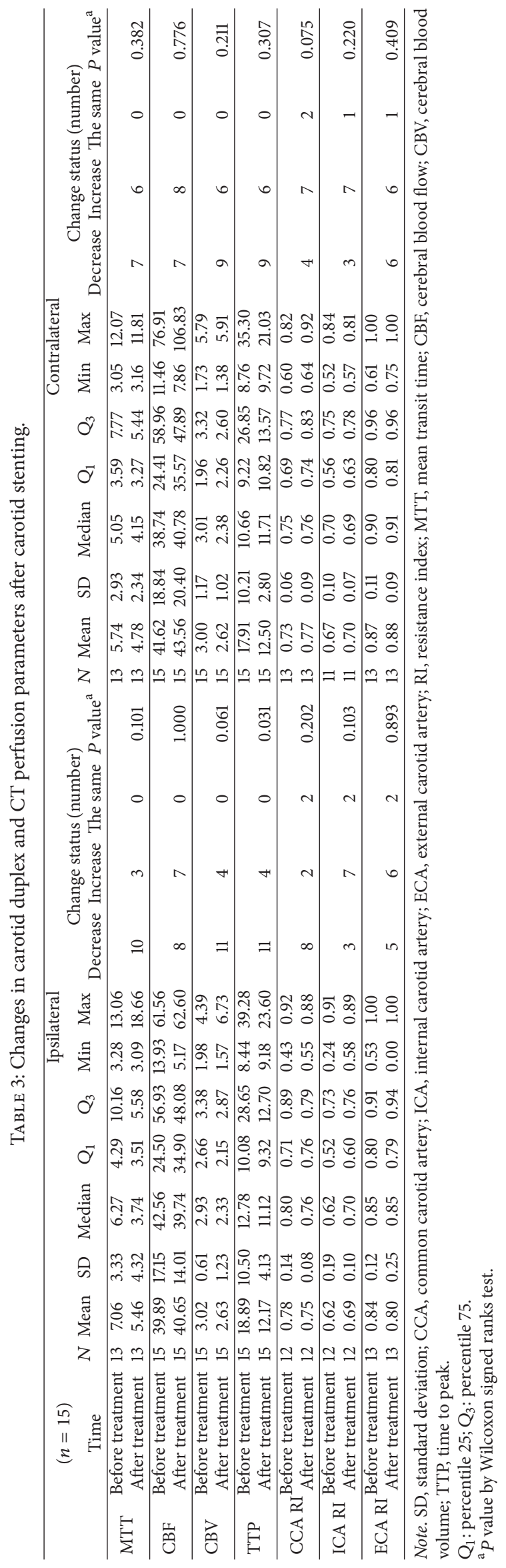




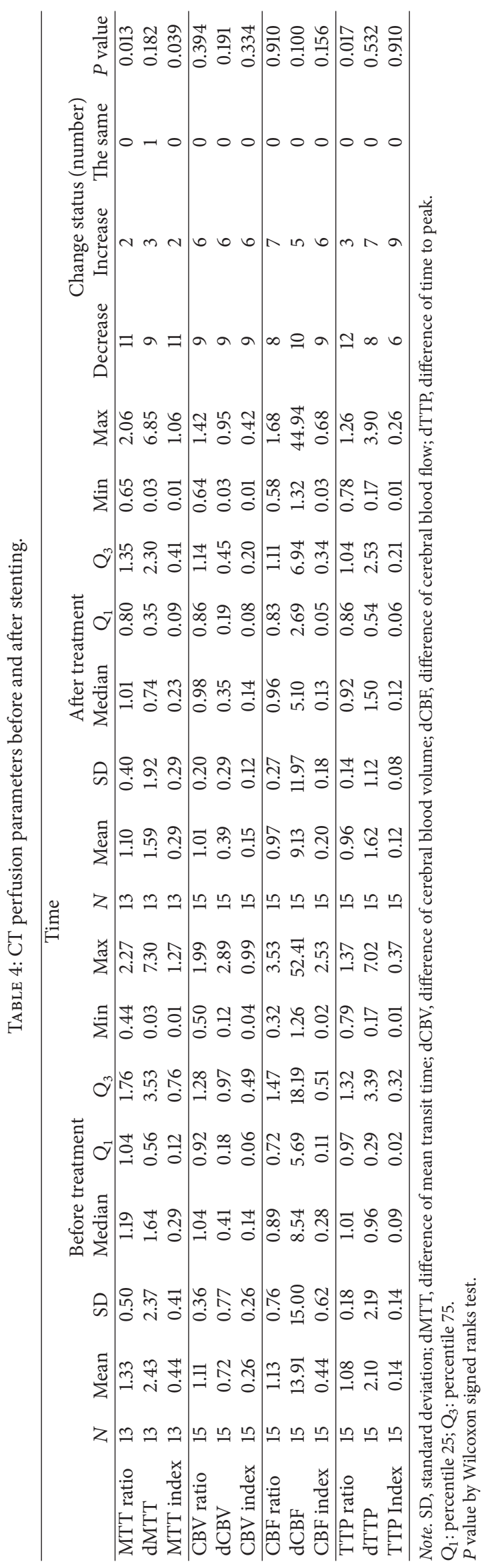


interpreted cautiously, and additional studies are necessary for confirming their applicability in various other conditions. Second, the carotid duplex and CT perfusion scans were conducted during peristenting phases. Although the immediate effect of stenting can be detected, the long-term cerebral perfusion status is yet to be investigated. Finally, all participants in this study were of Asian origin; therefore, the results can be generalized only to an Asian population.

\section{Conclusions}

The findings of our RI study on carotid ultrasounding and CT perfusion scanning can provide important clinical information in evaluating the perfusion status in patients receiving stenting, especially if the patient condition is not suitable for repeated CT perfusion examinations.

\section{Conflict of Interests}

The authors have no conflict of interests to declare.

\section{Authors' Contribution}

Chih-Ming Lin was responsible for collecting data and study design. Yu-Jun Chang was responsible for biostatistical analysis. Chi-Kuang Liu was responsible for data collection. Cheng-Sheng Yu was responsible for data analysis. Henry Horng-Shing Lu was responsible for study design and data analysis.

\section{Acknowledgments}

Special thanks are due to Ping-Yi Lin, Ph.D., of Chunghua Christian Hospital for her instructive comments. This project is partially supported by Ministry of Science and Technology and Big Data Research Center of National Chiao Tung University, Taiwan.

\section{References}

[1] P. Piñero, A. González, F. Moniche et al., "Progressive changes in cerebral perfusion after carotid stenting: a dynamic susceptibility contrast perfusion weighted imaging study," Journal of NeuroInterventional Surgery, vol. 6, no. 7, pp. 527-532, 2014.

[2] A. Trojanowska, A. Drop, T. Jargiello, J. Wojczal, and M. Szczerbo-Trojanowska, "Changes in cerebral hemodynamics after carotid stenting: evaluation with CT perfusion studies," Journal of Neuroradiology, vol. 33, no. 3, pp. 169-174, 2006.

[3] The European Carotid Surgery Trialists' Collaborative Group, "MRC European Carotid Surgery Trial: interim results for symptomatic patients with severe (70-99\%) or with mild (029\%) carotid stenosis," The Lancet, vol. 337, pp. 1235-1243, 1991.

[4] M. Kluytmans, J. van der Grond, K. J. van Everdingen, C. J. M. Klijn, L. J. Kappelle, and M. A. Viergever, "Cerebral hemodynamics in relation to patterns of collateral flow," Stroke, vol. 30, no. 7, pp. 1432-1439, 1999.

[5] Y. Suzuki, M. Nakajima, H. Ikeda, and T. Abe, "Evaluation of hyperacute stroke using perfusion computed tomography," Neurologia Medico-Chirurgica, vol. 45, no. 7, pp. 333-341, 2005.
[6] F. Miteff, C. R. Levi, G. A. Bateman, N. Spratt, P. McElduff, and M. W. Parsons, "The independent predictive utility of computed tomography angiographic collateral status in acute ischaemic stroke," Brain, vol. 132, no. 8, pp. 2231-2238, 2009.

[7] L. M. Jongen, J. Hendrikse, F. L. Moll, W. P. T. M. Mali, and H. B. van der Worp, "Cerebral perfusion affects the risk of ischemia during carotid artery stenting," Cerebrovascular Diseases, vol. 29, no. 6, pp. 538-545, 2010.

[8] K. M. Thierfelder, W. H. Sommer, A. B. Baumann et al., "Wholebrain CT perfusion: reliability and reproducibility of volumetric perfusion deficit assessment in patients with acute ischemic stroke," Neuroradiology, vol. 55, no. 7, pp. 827-835, 2013.

[9] Y. Duan, G. Li, Y. Yang et al., "Changes in cerebral hemodynamics after carotid stenting of symptomatic carotid artery," European Journal of Radiology, vol. 81, no. 4, pp. 744-748, 2012.

[10] L. E. Derchi, G. Leoncini, D. Parodi et al., "Mild renal dysfunction and renal vascular resistance in primary hypertension," American Journal of Hypertension, vol. 18, no. 7, pp. 966-971, 2005.

[11] G. H. Heine, M. K. Gerhart, C. Ulrich, H. Köhler, and M. Girndt, "Renal Doppler resistance indices are associated with systemic atherosclerosis in kidney transplant recipients," Kidney International, vol. 68, no. 2, pp. 878-885, 2005.

[12] G. H. Heine, B. Reichart, C. Ulrich, H. Köhler, and M. Girndt, "Do ultrasound renal resistance indices reflect systemic rather than renal vascular damage in chronic kidney disease?" Nephrology Dialysis Transplantation, vol. 22, no. 1, pp. 163-170, 2007.

[13] A. Kolonko, M. Szotowska, P. Kuczera, J. Chudek, and A. Wiecek, "Extrarenal factors influencing resistance index in stable kidney transplant recipients," Transplantation, vol. 96, no. 4, pp. 406-412, 2013.

[14] J. D. Van der Heyden, A. Waaijer, W. Van Es et al., "CT measurement of changes in cerebral perfusion in patients with asymptomatic carotid artery stenosis undergoing carotid stenting prior to cardiac surgery: 'proof of principle," EuroIntervention, vol. 6, no. 9, pp. 1091-1097, 2011.

[15] F. Gaudiello, V. Colangelo, F. Bolacchi et al., "Sixty-four-section CT cerebral perfusion evaluation in patients with carotid artery stenosis before and after stenting with a cerebral protection device," American Journal of Neuroradiology, vol. 29, no. 5, pp. 919-923, 2008.

[16] D. G. Nabavi, A. Cenic, R. A. Craen et al., "CT assessment of cerebral perfusion: experimental validation and initial clinical experience," Radiology, vol. 213, no. 1, pp. 141-149, 1999.

[17] L. Pourcelot, "Applications cliniques de l'examen Doppler transcutane," in Les Colloques de l'Institute National de la Sante et de la Recherche Medicale, vol. 34, pp. 213-240, INSERM, 1974. 


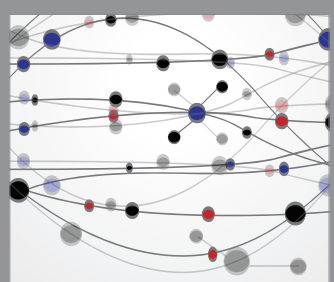

The Scientific World Journal
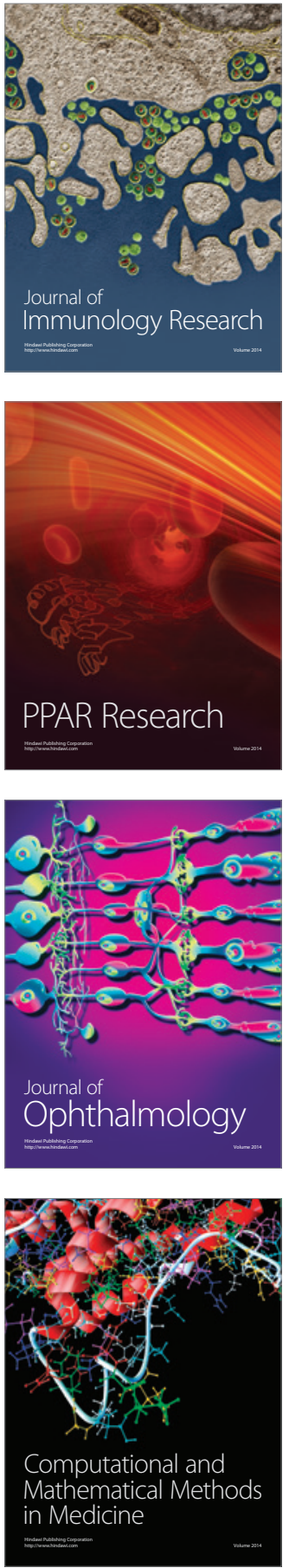



Gastroenterology Research and Practice

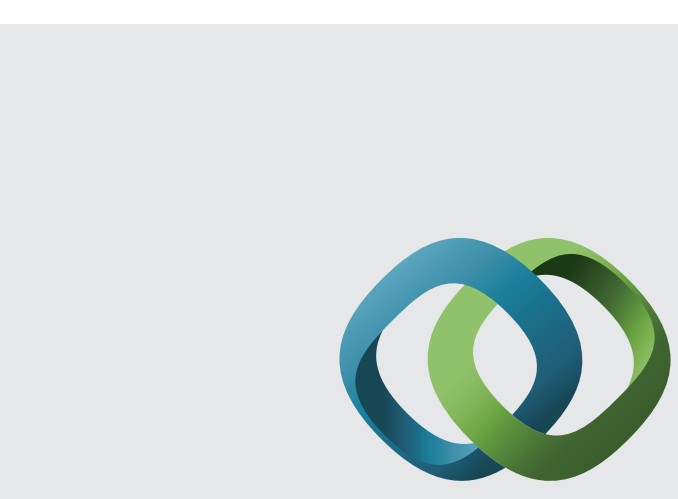

\section{Hindawi}

Submit your manuscripts at

http://www.hindawi.com
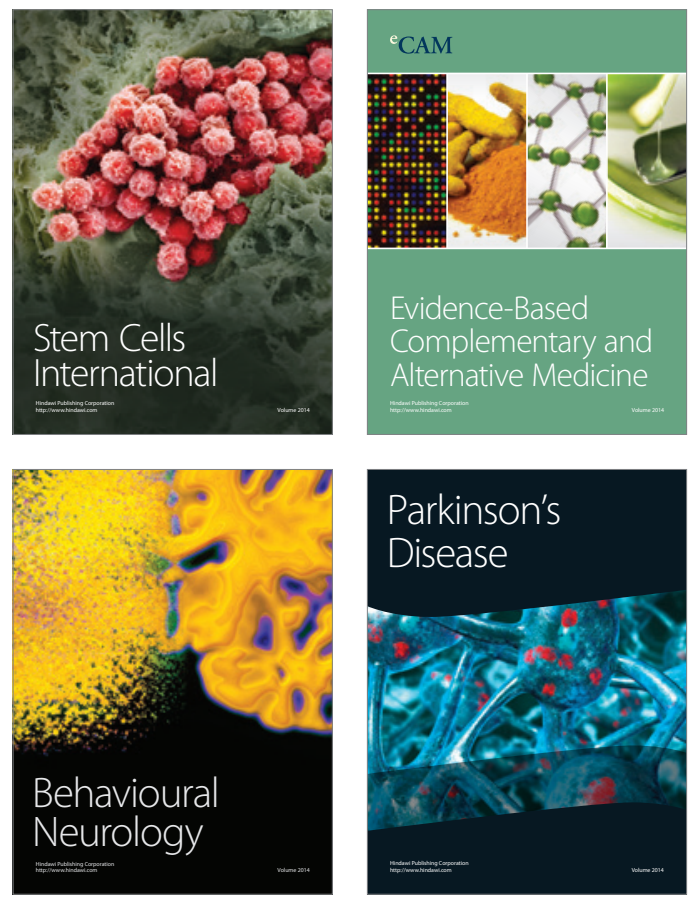
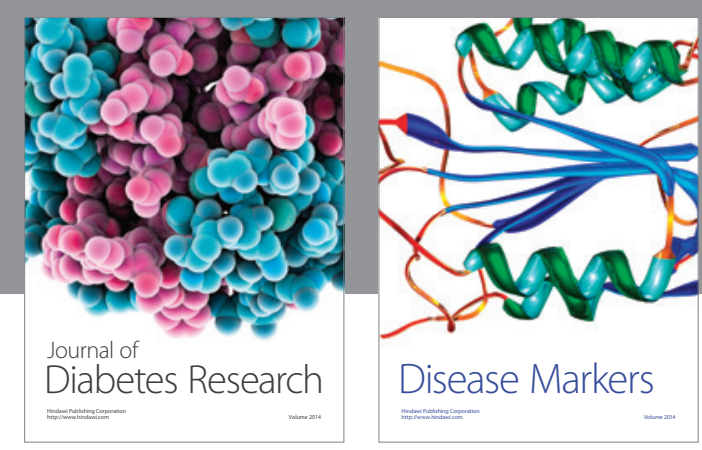

Disease Markers
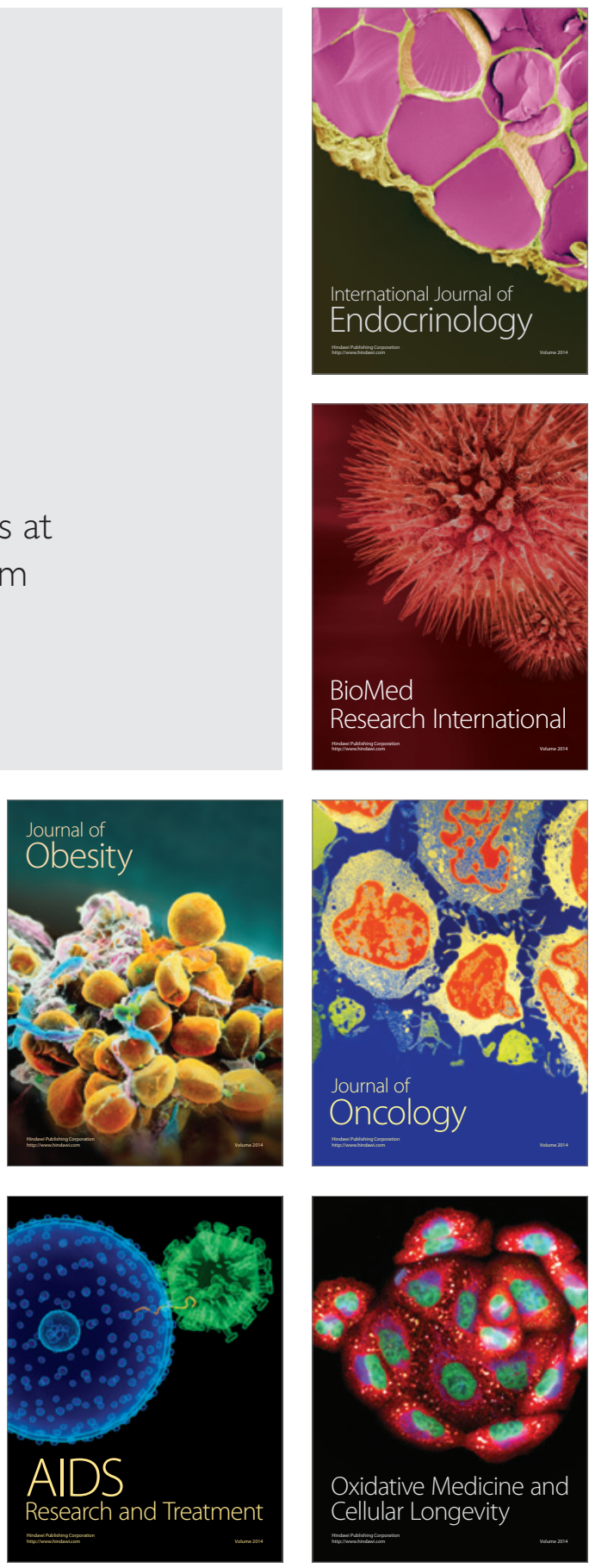